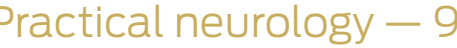 \\ Sudden limb weakness
}

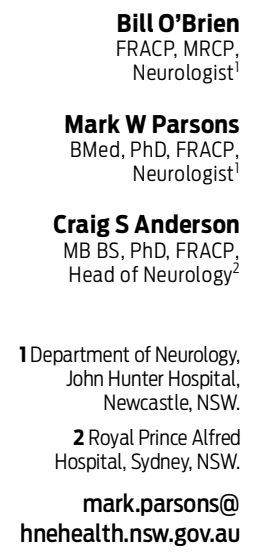

MJA 2012; 196: 572-577 doi: 10.5694/mjal2.10441

Previous article in this series "Upper limb tremor" in MJA 2012 196: 447-45

The grading system for recommendations in this article is described in MJA 2011; 195: 328

Series Editors

Craig $S$ Anderson MB BS, PhD, FRACP

Leo Davies MB BS, MD, FRACP

\section{Catherine's story}

The triage nurse in the emergency department (ED) of a metropolitan hospital contacted the hospital's acute stroke team at 10 am regarding Catherine, an 82-year-old woman who presented with a "probable stroke" with left-sided symptoms. Catherine was found collapsed on the kitchen floor by her daughter, who had visited at about $9.15 \mathrm{am}$. According to her daughter, Catherine had been up and doing her usual activities from $7 \mathrm{am}$ and was well when they had spoken on the telephone at $8 \mathrm{am}$. The electrocardiograph (ECG) monitor in the ambulance had shown Catherine to be in atrial fibrillation (AF) en route to the hospital. Apart from a history of treated hypertension, she had been in good health.

Soon after Catherine arrived at the ED, a rapid neurological examination undertaken by the neurology registrar showed that she was alert and obeying commands, but only with her right side. She was unable to look to the left (right gaze deviation) or appreciate finger movements on the left when presented as bilateral visual stimuli (left hemianopia). There was no movement in her left arm, which flopped back to her side when it was lifted up off the trolley. There was some movement in her left leg, but insufficient strength for her to raise it against gravity. She had reduced appreciation of touch sensation down her whole left side, most obvious in her left arm. The ECG monitor confirmed atrial fibrillation (heart rate, 100 beats/ $\mathrm{min}$ ) and her blood pressure was $180 / 90 \mathrm{mmHg}$. Apart from bruising on her left thigh from the fall, results of the rest of the physical examination were normal. This initial clinical assessment took only several minutes to complete.

\section{APPROACH TO THE PROBLEM}

\section{Brief focused neurological examination}

In Catherine's case, the history of sudden collapse and leftsided weakness, and associated focal neurological signs, is highly supportive of the diagnosis of acute stroke. However, once the registrar identified right gaze deviation and complete paralysis ("plegia") of the left arm and obtained a reliable time of onset of within the previous 2 hours, there was little more useful information to be gained from further physical examination that would effectively guide management.

A brief examination that focuses on detecting major neurological deficits enables rapid identification of the location and severity of any stroke. The most widely used structured grading system for assessing the severity of neurological deficit in the setting of acute stroke is the National Institutes of Health Stroke Scale (NIHSS). Assessment using the NIHSS takes less than 2 minutes, requires none of the paraphernalia of a full neurological examination (which is unnecessary in this situation) and is highly predictive of both short-term (in-hospital) and medium-term (at-home) functional outcomes (grade A evidence). ${ }^{1}$ Skilled use of the NIHSS requires only a few

\section{Summar}

- Stroke is a common neurological emergency and may occur in patients of all ages.

- Rapid assessment is crucial for patients with acute neurological symptoms suggestive of stroke because the opportunity for a positive outcome from thrombolytic treatment diminishes rapidly within the first few hours.

- Although plain non-contrast computed tomography of the brain is adequate to exclude haemorrhage and conditions such as malignancy, advanced multimodal imaging can be used to assist with decision making regarding the use of recombinant tissue plasminogen activator and mechanical clot-retrieval approaches without adding significant delay.

- Excellent outcomes are possible with the early use of reperfusion therapies, even when large areas of brain ischaemia are present, provided that there is evidence of potentially salvageable brain and that treatment can commence without unnecessary delay and hazard.

hours of training and accreditation (see http://www.americanheart.org and http://learn.heart.org/ihtml/application/ student/interface.heart2/nihss.html). The NIHSS includes a useful grading system for limb paresis that is based on "limb drift", which overcomes limitations of the traditional UK Medical Research Council grading system for muscle strength (0 for no movement to 5 for normal strength). The NIHSS assessment of limb strength requires the patient to raise an arm or leg off the bed. If he or she can raise it completely off the bed and hold it steady for 10 seconds, limb strength is considered normal and is scored 0 . If drift is noticed but the limb can be kept off the bed throughout, a score of 1 is given. If the limb falls to the bed within 10 seconds, a score of 2 is given. Inability to overcome gravity scores 3, and no movement of the limb scores 4 . If the patient is unable to adequately obey commands, the observation of spontaneous movement or drift (rapid return to the bed) after the assessor lifts the patient's limb in the air can allow adequate assessment of motor function.

\section{Appropriate use of investigations}

Clinically, Catherine had had a significant stroke and was within the time window for considering thrombolysis, so the next step was an urgent computed tomography (CT) scan to rule out haemorrhage. It is not uncommon to find no acute ischaemic changes on an initial CT scan in patients who have had an acute stroke because visible ischaemic changes usually lag several hours behind what is actually happening in the cerebral tissue. While magnetic resonance imaging can detect early cerebral ischaemia, this is less readily available than 
CT scanning for urgent cases in hospitals. New software that enables advanced multimodal CT imaging in patients who are being considered for thrombolysis is gaining popularity in assisting clinicians in their decision making regarding the use of thrombolysis (grade $C$ evidence). ${ }^{2,3}$

After Catherine's initial clinical assessment, she proceeded to non-contrast CT of her head, which showed no acute ischaemic changes. Perfusion CT (CTP) and CT angiogram (CTA) were then carried out, which confirmed that she had a mid-M1 segment occlusion of the right middle cerebral artery. Despite minimal infarcted cerebral tissue, the whole of the middle cerebral artery territory was at risk (ie, there was an ischaemic penumbra) (Box 1).

\section{PATHOPHYSIOLOGY OF CEREBRAL ISCHAEMIA}

Despite significant advances in a range of imaging modalities for investigating the brain and neurovascular tree over recent decades, the diagnosis of acute stroke is largely based on clinical examination, where the goal is to detect features of a sudden onset of focal neurological symptoms and signs of presumed vascular origin. As the anterior circulation has a large region of supply to the cerebral hemispheres, it is most often involved in both ischaemic and haemorrhagic strokes.

Typical features of major strokes include varying degrees and/or combinations of paresis, dysphasia, dysarthria, visual field loss, and disturbance of hemisensory and visuospatial perception. While strokes rarely cause sudden cardiac arrest and death, they can result in an immediate loss of, or rapid deterioration in, a person's level of consciousness. This can occur because of subarachnoid haemorrhage, mass effect of intracerebral haemorrhage producing cerebral midline or brainstem shift, direct ischaemia of the brainstem (basilar artery occlusion), or generalised seizures triggered by cerebral ischaemia or trauma.

In contrast, minor strokes can be subtle neurological events that may stutter or evolve over hours or days (Box 2 ). Thus without a history of sudden onset (from the patient or a reliable witness) and/or the presence of atypical, "positive" or inconsistent features, the likelihood of an underlying alternative diagnosis (ie, stroke mimic) is increased (Box 3). In the past, a cut-point of 24 hours was arbitrarily chosen as the duration of neurological symptoms used to separate the diagnosis of transient ischaemic attack from acute stroke in clinical and epidemiological research, but this is no longer clinically significant.

Most strokes (>80\%) are ischaemic and the remainder are due to haemorrhage, which can easily be differentiated on a non-contrast CT scan of the brain. Ischaemic stroke is caused by acute occlusion (clot) of an artery that leads to an immediate reduction in blood flow in the corresponding cerebrovascular territory. The size and site of the occlusion, and the efficiency of compensatory collateral blood flow, determine the extent of impaired blood flow and resulting neurological symptoms from at-risk brain tissue (the ischaemic penumbra [brain tissue that remains potentially salvageable but where blood supply is reduced
1 Multimodal computed tomography (CT) and magnetic resonance imaging (MRI) images of Catherine's brain before and after treatment with recombinant tissue plasminogen activator
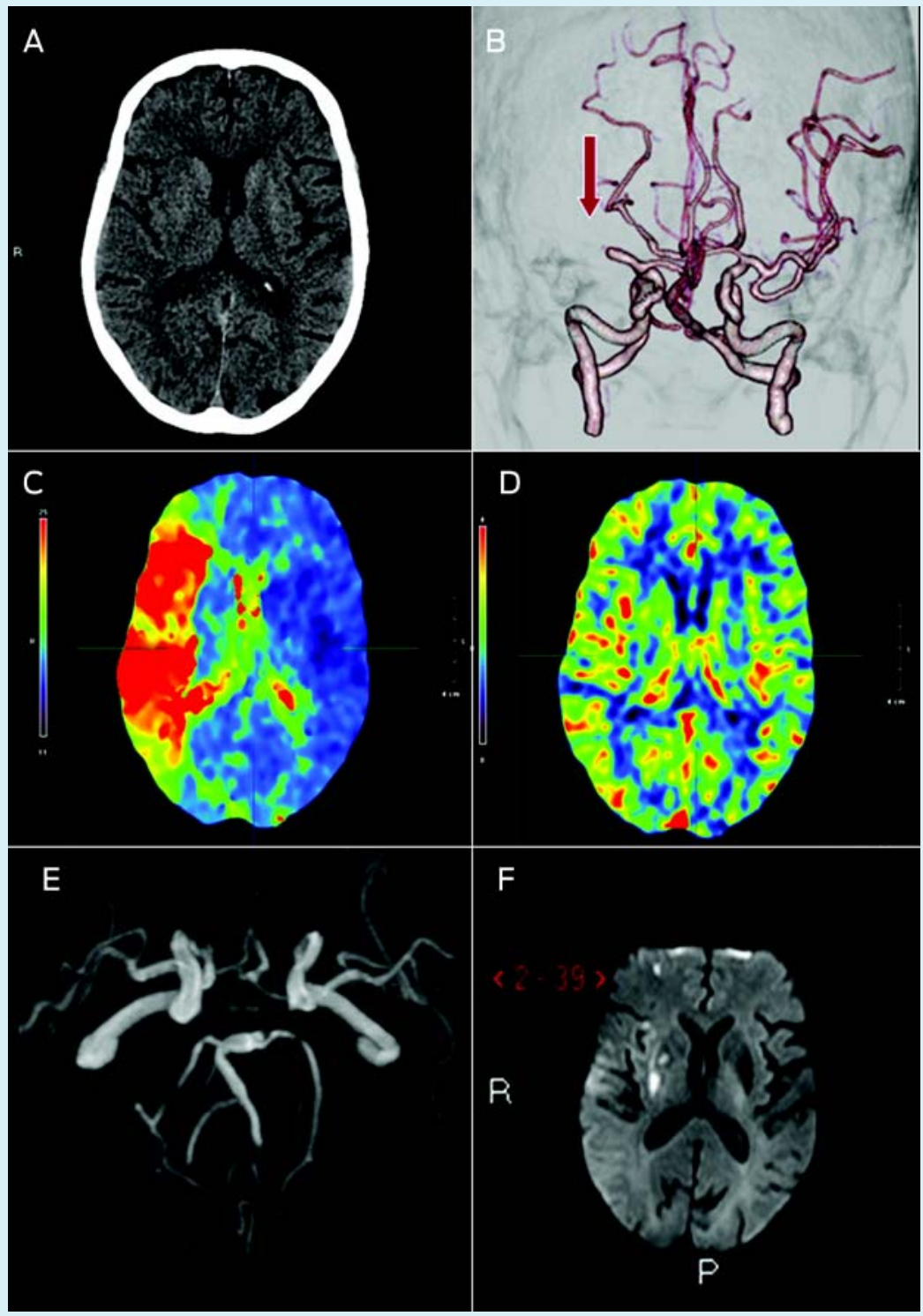

A: Non-contrast CT showing no acute changes, as is often the case in acute stroke.

$B$ : CT angiogram showing occlusion of the mid-M1 segment of the middle cerebral artery (arrow) with no distal flow.

C: CT perfusion time-to-peak map showing a significant delay in contrast flow (red area) affecting the right hemisphere, indicating that the entire territory is at risk of infarction without timely reperfusion.

D: CT perfusion mean-transit-time map showing that, despite the significant perfusion deficit on the time-to-peak map, cerebral blood flow in the right hemisphere is preserved (with no obvious asymmetry compared with the left hemisphere), which suggests that as yet little hypoperfused tissue has progressed to established infarction.

E: Magnetic resonance angiography 24 hours after treatment showing complete recanalisation of the middle cerebral artery.

F: Diffusion-weighted MRI (a very sensitive measure of acute infarction) 24 hours after treatment showing a few small acute infarcts (high signal in right hemisphere), but essentially complete salvage of the territory that had been at risk.

Perfusion brain imaging (with CT or MRI) involves use of an intravenous contrast bolus followed by serial imaging of the brain over about 1 minute, with tracking of the contrast as it flows through the vascular bed, allowing assessment of cerebral blood flow, cerebral blood volume, time to peak and mean transit time. Delayed contrast transit on time-to-peak or mean-transit-time maps is most often used to identify tissue that is at risk and areas where severely reduced cerebral blood volume (or blood flow) represents irreversibly injured tissue. 
2 Minor stroke syndromes that require particular attention in assessment

\begin{tabular}{|c|c|c|c|}
\hline Symptoms & $\begin{array}{l}\text { Neurological } \\
\text { syndrome }\end{array}$ & $\begin{array}{l}\text { Pathological } \\
\text { condition }\end{array}$ & Clinical examination tips \\
\hline $\begin{array}{l}\text { Confusion, } \\
\text { speech not } \\
\text { making sense }\end{array}$ & $\begin{array}{l}\text { Isolated fluent } \\
\text { dysphasia } \\
\text { (receptive or } \\
\text { Wernicke } \\
\text { aphasia) }\end{array}$ & $\begin{array}{l}\text { Lesion of the } \\
\text { temporal-parietal } \\
\text { region due to an } \\
\text { embolic occlusion of } \\
\text { the terminal branch } \\
\text { of the left middle } \\
\text { cerebral artery* }\end{array}$ & $\begin{array}{l}\text { Check premorbid status to ascertain that there has been a sudden change in speech, } \\
\text { memory or behaviour } \\
\text { - Test receptive language by asking the patient to follow sequentially simple commands } \\
\text { ("open your mouth", "poke out your tongue") and then more complex commands ("close } \\
\text { your eyes and open your mouth", "put your left hand on your right ear") } \\
\text { - Test the visual fields to determine whether there is any deeper ischaemia involving the } \\
\text { visual radiation }\end{array}$ \\
\hline $\begin{array}{l}\text { Confusion, acting } \\
\text { strangely }\end{array}$ & $\begin{array}{l}\text { Isolated } \\
\text { visuospatial } \\
\text { perceptive loss }\end{array}$ & $\begin{array}{l}\text { Lesion of the } \\
\text { temporal-parietal } \\
\text { region due to an } \\
\text { embolic occlusion of } \\
\text { the terminal branch } \\
\text { of the right middle } \\
\text { cerebral artery }\end{array}$ & $\begin{array}{l}\text { Check premorbid status to ascertain that there has been a sudden change in memory or } \\
\text { behaviour } \\
\text { Check whether the patient can perform complex motor tasks with the left hand (eg, } \\
\text { demonstrate how to salute, hold a cigarette or comb hair) } \\
\text { Check sensory perception in the left hand (eg, sensory inattention or neglect, and } \\
\text { recognition of shapes, textures and numbers) } \\
\text { Check for dressing dyspraxia }\end{array}$ \\
\hline $\begin{array}{l}\text { Sudden blurred } \\
\text { vision }\end{array}$ & $\begin{array}{l}\text { Isolated visual } \\
\text { field defect }\end{array}$ & $\begin{array}{l}\text { Lesion of the } \\
\text { occipital lobe due to } \\
\text { a distal embolic } \\
\text { occlusion of an } \\
\text { occipital artery }\end{array}$ & $\begin{array}{l}\text { Pay careful attention to confrontation testing of the visual fields to check for a } \\
\text { quadrantanopia or visual inattention }\end{array}$ \\
\hline $\begin{array}{l}\text { Acting strangely } \\
\text { or out of } \\
\text { character }\end{array}$ & $\begin{array}{l}\text { Isolated frontal } \\
\text { lobe deficit }\end{array}$ & $\begin{array}{l}\text { Intracerebral } \\
\text { haemorrhage of the } \\
\text { frontal lobe }\end{array}$ & $\begin{array}{l}\text { During examination of orientation and cognition, pay particular attention to responses for } \\
\text { current affairs (eg, recent news events), perception and reasoning (eg, interpreting English } \\
\text { proverbs such as "too many cooks spoil the broth"), and processing of complex mental } \\
\text { tasks (eg, the "serial sevens" task) }\end{array}$ \\
\hline $\begin{array}{l}\text { Sudden severe } \\
\text { headache, like } \\
\text { being hit on the } \\
\text { head }\end{array}$ & $\begin{array}{l}\text { Sudden } \\
\text { unaccustomed } \\
\text { severe headache }\end{array}$ & $\begin{array}{l}\text { Subarachnoid } \\
\text { haemorrhage }\end{array}$ & $\begin{array}{l}\text { - Check for nuchal rigidity (ie, neck stiffness) } \\
\text { - CT head scan within } 48 \text { hours of symptoms can detect blood in }>95 \% \text { of cases } \\
\text { - Lumbar puncture in cases of delayed presentation }\end{array}$ \\
\hline $\begin{array}{l}\text { Sudden vertigo, } \\
\text { nausea and } \\
\text { vomiting }\end{array}$ & $\begin{array}{l}\text { Acute disturbance } \\
\text { of vestibular } \\
\text { pathways }\end{array}$ & $\begin{array}{l}\text { Small cerebellar or } \\
\text { brainstem lesion }\end{array}$ & $\begin{array}{l}\text { If the patient is extremely disabled and unable to stand or walk, an urgent CT head scan } \\
\text { can exclude major ischaemia or haematoma with mass effect that is potentially relieved } \\
\text { by decompressive surgery } \\
\text { Check for additional neurological signs associated with the vestibular disturbance to } \\
\text { indicate involvement of the brainstem (eg, limb paresis, dysphagia, or disturbance of } \\
\text { sensation over the face, trunk or limbs) or cerebellum (eg, limb or trunk ataxia) } \\
\text { Position changing or vertical gaze nystagmus indicates a central (brainstem) cause }\end{array}$ \\
\hline
\end{tabular}

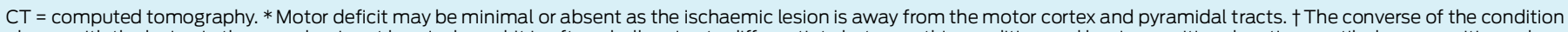

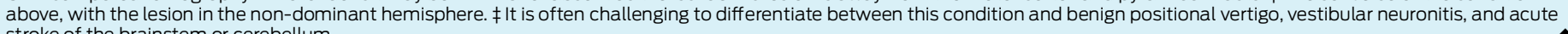
stroke of the brainstem or cerebellum.

to a level that unless restored will progress to infarction]) and/or dead brain tissue (the ischaemic core). ${ }^{4}$

Early spontaneous recanalisation may occur from the normal endogenous release of tissue plasminogen activator (tPA), a serine protease of the fibrinolytic system that converts the zymogen plasminogen into the active protease plasmin, which leads to cleavage of fibrin and dissolving of the newly formed thrombin clot. For most patients, however, and particularly those with large clots, this natural physiological function is inadequate to avoid the outcome of neurological disability from resulting cerebral infarction. Modern management of acute ischaemic stroke is, therefore, aimed at intervening as early as possible after symptom onset to facilitate rapid vessel recanalisation and reperfusion to preserve cerebral function and promote a good outcome.

\section{MANAGEMENT}

Acute stroke can be treated rapidly and effectively in an ED with the basic clinical skills that all physicians have. This case highlights the crux of acute intervention in ischaemic

stroke - reperfusion of the ischaemic penumbra. ${ }^{5}$ Intravenous recombinant tissue plasminogen activator (rtPA) is the only treatment licensed by the TGA for treatment of ischaemic stroke, and in a substantial number of patients will result in effective dissolution of the clot.

Current imaging guidelines rely on non-contrast CT but advances in technology are now at a point where a more accurate assessment can be made with multimodal imaging. Treatment is effective but it has limitations (less than $30 \%$ of patients with proximal occlusions [eg, terminal internal carotid or origin middle cerebral artery] will have successful dissolution of the clot). As more powerful thrombolytics become available and intra-arterial approaches gain a foothold, patient outcomes will undoubtedly be advanced further.

\section{Thrombolytic treatment}

FINAL DIAGNOSIS Acute ischaemic stroke
There is compelling evidence to support the routine use of intravenous rtPA in patients with acute ischaemic stroke when carried out in experienced centres with adequate expertise available (grade A evidence). ${ }^{6}$ 
Syncope

- Sudden collapse with brief loss of consciousness (few seconds) and full recovery

- Preceding "dizziness", palpitations or chest pain is common

- Situational specificity is common (eg, symptoms triggered by transfer to stand, micturition or showering)

- Witness report of patient being pale, sweating and pulseless during event

- Brief jerking movements may occur

\section{Seizures}

- The generalised tonic-clonic convulsive episode may not be witnessed, and the patient may first present with postictal drowsiness or confusion and variable degrees of hemiparesis or dysphasia, which recovers over hours with normal brain scan results

- Persistent jerking or twitching movements and/or slow recovery that is disproportionate to the size of any lesion identified by brain imaging

- Recurrent transient interruptions of behaviour, speech and alertness, with or without warning auras of nausea or altered bodily sensation (suspect temporal lobe seizures)

\section{Migraine}

- History of recurrent headaches and variable degrees of visual auras, and nausea, vomiting and debility during events

- Non-specific sensory symptoms in an otherwise well young adult (particularly in women)

\section{Delirium}

- Variable and fluctuating degrees of confusion without focal neurological signs

\section{Benign positional vertigo}

- History of recurrent attacks of vertigo, which may be triggered by sudden movements of the head

- Vertigo, nausea and vomiting are the prominent symptoms

- Examination is difficult in patients with severe disturbance of their vestibular system - unable to move in any direction or stand
Functional weakness, gain or other neurological disturbance

- Variable and inconsistent physical findings in a younger adult

- Bizarre pattern of symptoms and signs which does not conform to neuroanatomical location

- Inappropriate emotional response

Drug toxicity

- Suspicion of abnormal reaction to life events or stress

- Nystagmus and ataxia (can be due to alcohol)

- Reduced level of consciousness and variable limb paresis (can be due to amphetamines and heroin)

- Overdose toxicity (eg, anticonvulsant, tricyclic antidepressant or lithium overdose)

\section{Transient global amnesia}

- Temporary impairment of short-term memory (several hours), with intact immediate recall and long-term memory and other cognitive functions, in an otherwise lucid and alert person

- No focal motor or other neurological signs

- No recent seizure or head injury

- On recovery, patient has some recall of the disturbance

Bell palsy

- Lower motor neurone facial paresis with loss of elevation of the eyebrow due to paresis of the frontalis muscle

- Variable pain over the ear

- Vesicles in the auditory canal may be present (indicating varicellazoster infection)

Systemic illness in a patient with an existing major stroke deficit

- Due to the existing injury, any disturbance of metabolism or circulation may worsen the deficit and increase the possibility of a recurrent stroke

- Seizures are common in patients with prior stroke, and these may not have tonic-clonic features

- Increasing drowsiness and loss of consciousness without alteration in motor signs may indicate drug toxicity, metabolic disturbance or seizures
While the role of rtPA in acute ischaemic stroke has been debated, particularly within the emergency medicine community, current guidelines from the National Stroke Foundation (which are endorsed by the Australasian College for Emergency Medicine) and licensing by the Therapeutic Goods Administration reflect the evidence for use of rtPA up to 4.5 hours after symptom onset. ${ }^{7}$ Given that the chance of benefit is greatest when rtPA is used early, because ischaemia causes at-risk brain tissue to rapidly progress to irreversible infarction, the "time is brain" motto is most pertinent in defining systems of care for assessing patients who are potentially eligible for thrombolytic treatment. With a rapid chain of referral, thrombolysis rates of over $20 \%$ can be achieved (grade $\mathrm{C}$ evidence). ${ }^{8}$ Although there are risks of bleeding associated with rtPA, the most serious of which is intracerebral haemorrhage that is large enough to produce neurological deterioration, the risks and benefits, when given within the current time window, favours treatment, and this translates into one fewer patient dead or dependent for every 10 patients treated. ${ }^{6}$ Although the patient characteristics that predict the least to gain or the greatest hazard from rtPA have not been adequately defined, rtPA should be used with caution in certain situations (grade C evidence) (Box 4). It is clear that the benefit of rtPA is very much dependent on there being a clear time of onset of symptoms. This is not possible in up to one-fifth of patients. If a patient cannot provide a reliable history, then the onset of the stroke must be taken as the time when he or she was last seen well. This can be particularly challenging when the patient's symptoms are present on waking ("wake-up stroke"), in which case the onset time must be taken as when he or she was last known to be well before going to sleep.

While inclusion and exclusion screening questionnaires are readily available for patients who are being considered for thrombolysis, the main practical issue of interest is an assessment of the risk of haemorrhage. ${ }^{7}$ Although blood tests, including tests of coagulation profile, are routinely undertaken for patients who have had a stroke, the results are usually normal in those who have previously been well and should not hold up the administration of rtPA (grade B

\footnotetext{
4 Situations where intravenous recombinant tissue plasminogen activator should be used with caution

- Large area of infarction on initial non-contrast computed tomography scan, indicating that time since onset is likely to be $>4.5$ hours

- Evidence of proximal vessel (terminal internal carotid ["carotid T"]) occlusion - in which case the patient may benefit from mechanical clot extraction procedures

- Patient is older than 80 years

- Patient is taking warfarin and has an international normalised ratio $>1.7$, or is taking other therapeutic anticoagulants

- Significant trauma from fall at time of stroke onset
} 


\section{Aspects of stroke that should be reviewed at a follow-up clinic}

What is the probable aetiology of the stroke?

- Large vessel disease - degree of extracranial or intracranial atheroma

- Small vessel disease - hypertension

- Cardioembolism - atrial fibrillation or poor cardiac function

- Dissection of cerebral vessel

- Rare forms of stroke

Have all necessary investigations been undertaken and have the results been reviewed?

Check results of transthoracic or transoesophageal echocardiography, carotid ultrasound, angiography, haematological and biochemical tests

Have all appropriate stroke prevention strategies been implemented?

Blood pressure lowering in all patients, aiming for a target systolic level of $<140 \mathrm{mmHg}$

- Cholesterol lowering in all patients who have had ischaemic stroke

- Aspirin or other antithrombotic agent in all patients who have had ischaemic stroke unless there is a clear contraindication

- Warfarin anticoagulation in all patients who have had a stroke with a clear cardioembolic source

- Carotid endarterectomy or stenting in patients who have had ischaemic stroke due to ipsilateral high-grade carotid stenosis

Have the patient and family members obtained a reasonable understanding of the stroke?

- Simple descriptors include relating the size of the stroke to that of a fruit (eg, apple or plum) and drawing an analogy between the size and location of related blood vessels and the trunk, branches and leaves of a tree

Are there any complications of the stroke?

- Depression - in up to 30\% of patients, with half requiring treatment

- Emotionalism - easy crying that is readily treated with selective serotonin reuptake inhibitors

- Shoulder pain - due to subluxation or capsulitis resulting from a flaccid arm

- Falls - due to poor mobility, environmental hazard or impulsiveness

- Cognitive impairment - may be subtle and may present as apathy, slowness, forgetfulness and "not being right"

- Behavioural change - relating to poor adjustment to altered circumstances

Seizures - in $5 \%$ of patients

- Central pain syndromes - very difficult to manage with conventional medical treatments, including analgesics, antidepressants and anticonvulsants

Are there any outstanding matters to address?

- Resumption of driving after 1 month in those without residual physical, cognitive or visual impairment

- Resumption of sex

- Request for continuation of sick leave

- Completion of documents necessary for income protection insurance, workers compensation or pension

\section{Does anything crop up during discussion and provision of feedback?}

- As stroke is a common life-threatening illness with high chances of major ongoing disability, patients benefit immensely from receiving positive feedback that: the stroke was "minor"; further investigations are not necessary; and they have recovered, or are doing, well

evidence). However, in patients on warfarin therapy, rtPA should be avoided or used with caution if the international normalised ratio is above 1.7 . $^{7}$

\section{Further history-taking}

Premorbid functioning is an important aspect of enquiry in older patients, as the balance of benefits and risks of thrombolysis is not as well defined in older patients as it is in younger adults. As such, clinicians can be presented with an ethical dilemma over the appropriate use of thrombolysis - a complex and risky treatment - in those older than 80 years. ${ }^{9}$ A global assessment of premorbid functioning can be made with specific enquiry regarding the patient's ability to manage daily affairs, such as whether he or she is able to do his or her own shopping. Another useful guide of function is how long a family member would be prepared to leave the person on their own - for example, during the day or overnight. Age alone does not preclude the use (and potential benefits) of thrombolysis but an accurate assessment of premorbid functioning helps guide decision making (grade B evidence). ${ }^{7}$ As a general rule, patients of any age who have had a large "completed" stroke with large area of cerebral infarction before treatment do poorly irrespective of treatment.

When the neurology registrar enquired about Catherine's premorbid functioning, her daughter described her as being independent in all daily activities, including shopping, and cognitively sharp without memory problems.

On review of Catherine's eligibility for rtPA, she was found to be in AF, which had not been previously diagnosed. (Undiagnosed AF is not uncommon in patients who have had a stroke, because AF is often asymptomatic and intermittent before becoming persistent, with common triggers including alcohol intake, acute illness and obstructive sleep apnoea.) She had not been taking any antithrombotic treatment and had no history of bleeding. Her blood pressure was less than $185 / 110 \mathrm{mmHg}$ (the cutoff for treatment with rtPA).

A frank discussion with Catherine and her daughter involved outlining the benefits and risks of thrombolysis, including the potential for symptomatic haemorrhage. Based on the additional information gained from advanced CT imaging, it was very likely that Catherine would develop major infarction and have significant long-term disability if rapid recanalisation and perfusion of the at-risk cerebral hemisphere did not occur. Catherine and her daughter agreed to proceed with the recommended decision of intravenous rtPA treatment, which was given as per the standard protocol ( $0.9 \mathrm{mg} / \mathrm{kg}$ total dose; $10 \%$ bolus and the remainder by infusion over 1 hour).

Catherine was transferred to the hospital's stroke unit for close monitoring of her vital and neurological signs over the next 24 hours. Two hours after completion of the rtPA infusion, a transcranial colour doppler ultrasound showed return of normal flow in the right middle cerebral artery. She could look to the left and had antigravity movement in her left arm and leg.

A "Lazarus-like" response such as this occurs in about one in 10 rtPA-treated patients; it results from there being minimal early infarction before treatment combined with early reperfusion of a large region of ischaemic brain. Thus, in the presence of a pattern of small infarct core and large ischaemic penumbra on advanced CT imaging (or magnetic resonance imaging), the Lazarus effect is quite common, although it is dependent on the rtPA being successful in rapidly dissolving the occlusive clot (grade $\mathrm{C}$ evidence) ${ }^{10}$

On review the next day, Catherine had no focal neurological deficit, and complete reperfusion and minimal infarction were shown on MRI (Box 1). She was commenced on longterm warfarin therapy. She was reviewed by allied health staff to check on her cognition, mobility and self-care activities, to ensure that it was safe for her to be discharged home. On Day 5, she was back to her premorbid level of functioning and was discharged.

Five weeks later, Catherine was followed up in an outpatient stroke clinic. She had maintained an adequate level of anticoagulation according to weekly testing. 


\section{FACT OR FICTION?}

Fact: It is true that patients treated with rtPA can make a full and complete recovery from acute ischaemic stroke.

Fiction: It is not true that it takes too long to undertake an accurate neurological examination in patients presenting with acute stroke symptoms.

\section{Other interventions}

Other proven interventions in the management of acute stroke include admission to and management in a stroke unit with the associated nursing and allied health expertise and, in patients with ischaemic stroke, commencement of aspirin therapy (100-150 mg daily) within 48 hours of symptom onset (grade A evidence). ${ }^{7}$ These should be considered for all patients who have had an ischaemic stroke, regardless of whether they are eligible for rtPA.

\section{Follow up}

An outpatient stroke clinic allows the opportunity to: undertake outstanding investigations and review the results; check that patients are recovering satisfactorily, adjusting to residual disability, and adhering to lifestyle modifications (particularly cessation of smoking) and secondary stroke prevention strategies; review continuity of follow-up rehabilitation or support services; review communication with the patient's general practitioner; check that the patient and his or her family have a reasonable understanding of stroke and its aetiology; and check that there are no major complications or comorbid consequences of the stroke event (Box 5).

Competing interests: No relevant disclosures.

Provenance: Commissioned; externally peer reviewed.

1 Kasner SE. Clinical interpretation and use of stroke scales. Lancet Neurol 2006; 5: 603-612.

2 Muir KW, Buchan A, von Kummer R, et al. Imaging of acute stroke. Lancet Neurol 2006; 5: 755-768.

3 Parsons MW. Perfusion CT: is it clinically useful? Int J Stroke 2008; 3: 41-50.

4 Donnan GA, Fisher M, Macleod M, Davis SM. Stroke. Lancet 2008; 371: $1612-1623$.

5 Rha JH, Saver JL. The impact of recanalization on ischemic stroke outcome: a meta-analysis. Stroke 2007; 38: 967-973.

6 Lees KR, Bluhmki E, von Kummer R, et al. Time to treatment with intravenous alteplase and outcome in stroke: an updated pooled analysis of ECASS, ATLANTIS, NINDS, and EPITHET trials. Lancet 2010; 375: 1695-1703.

7 National Stroke Foundation. Clinical guidelines for stroke management 2010. Melbourne: NSF, 2010. http://www.strokefoundation.com.au/news/welcome/ clinical-guidelines-for-acute-stroke-management (accessed Apr 2012).

8 Quain DA, Parsons MW, Loudfoot AR, et al. Improving access to acute stroke therapies: a controlled trial of organised pre-hospital and emergency care. Med J Aust 2008; 189: 429-433.

9 Mishra NK, Ahmed N, Andersen G, et al. Thrombolysis in very elderly people: controlled comparison of SITS International Stroke Thrombolysis Registry and Virtual International Stroke Trials Archive. BMJ 2010; 341: c6046.

10 Parsons M, Spratt N, Bivard A, et al. A randomized trial of tenecteplase versus alteplase for acute ischemic stroke. N Engl J Med 2012; 366: 1099-1107. 